\title{
Free-breathing 3D cardiac function with accelerated magnetization transfer prepared imaging
}

\author{
Eric M Schrauben ${ }^{1 *}$, Oliver Wieben ${ }^{1,2}$, Kevin M Johnson ${ }^{1}$ \\ From 17th Annual SCMR Scientific Sessions \\ New Orleans, LA, USA. 16-19 January 2014
}

\section{Background}

3D cardiac MRI has long held promise for improved heart coverage, higher resolution, and reduced sensitivity to poor breath-hold reproducibility. However, its use has been limited by reduced blood pool to myocardium contrast for spoiled and balanced steady-state free precession (bSSFP) implementations. T2-preparation techniques [1] are capable of increasing contrast but are unfortunately limited by lengthy preparation periods and resulting scan inefficiencies. In this work, we develop a paradigm for high contrast 3D cardiac function that relies on the alternative use of magnetization transfer (MT) preparation [2] combined with accelerated 3D spoiled gradient echo imaging (SPGR).

\section{Methods}

An off-resonance RF pulse was interleaved with wholeheart, respiratory gated 3D radial SPGR sampling [3]. Simulations and phantom scans were performed to optimize MT saturation (power, off-resonance, and frequency). Phantom scans utilized $4 \%$ agar, fat, and doped water. After optimization, initial volunteer images were collected on a clinical $1.5 \mathrm{~T}$ system (HDx, GE, Waukesha, WI) using: FOV $=64 \times 32 \times 32 \mathrm{~cm} 3,2.0 \mathrm{~mm}$ isotropic spatial resolution, TR/TE1/TE2 $=5.6 / 1.32 / 3.32$ $\mathrm{ms}, \alpha=4^{\circ}$, free-breathing: scan time $=10 \mathrm{~min}, 50 \%$ acceptance window (bellows), number of projections = 39,000 . In-vivo experiments utilized a $1600^{\circ}, 20 \mathrm{~ms}$ Hamming-windowed Sinc pulse applied every 10 TRs. This pulse was applied at $210 \mathrm{~Hz}$ off-resonance provid-

ing some fat-saturation. In addition, two full echoes (TE1 and TE2) at $\pm 62.5 \mathrm{kHz}$ were added to further remove fat signal while increasing SNR of water images. Twenty cardiac time frames were reconstructed using iterative soft thresholding of temporal differences with a spatial wavelet transform.

\section{Results}

Figure 1 shows images from phantom scans for a sweep of MT off-resonance frequencies and demonstrates the potential for simultaneous suppression of muscle (agar) and fat. In-vivo results are presented in Figure 2 for two reformats: vertical long axis in end-systole and end-diastole (left) and an end-systolic base to apex short axis stack (right). Excellent blood pool to myocardium contrast and fat suppression are observed. Isotropic spatial resolution allows for retrospective whole-heart reformats in any orientation.

\section{Conclusions}

The feasibility of a novel whole-heart functional cardiac acquisition using MT preparation with isotropic spatial resolution in a clinically reasonable scan time is presented. Further studies on optimization of acquisition parameters, including off-resonance frequency, number of projections, and acquired spatial resolution, will improve the applicability of the sequence for clinical situations.

\section{Funding}

NIH grant 2R01HL072260. 

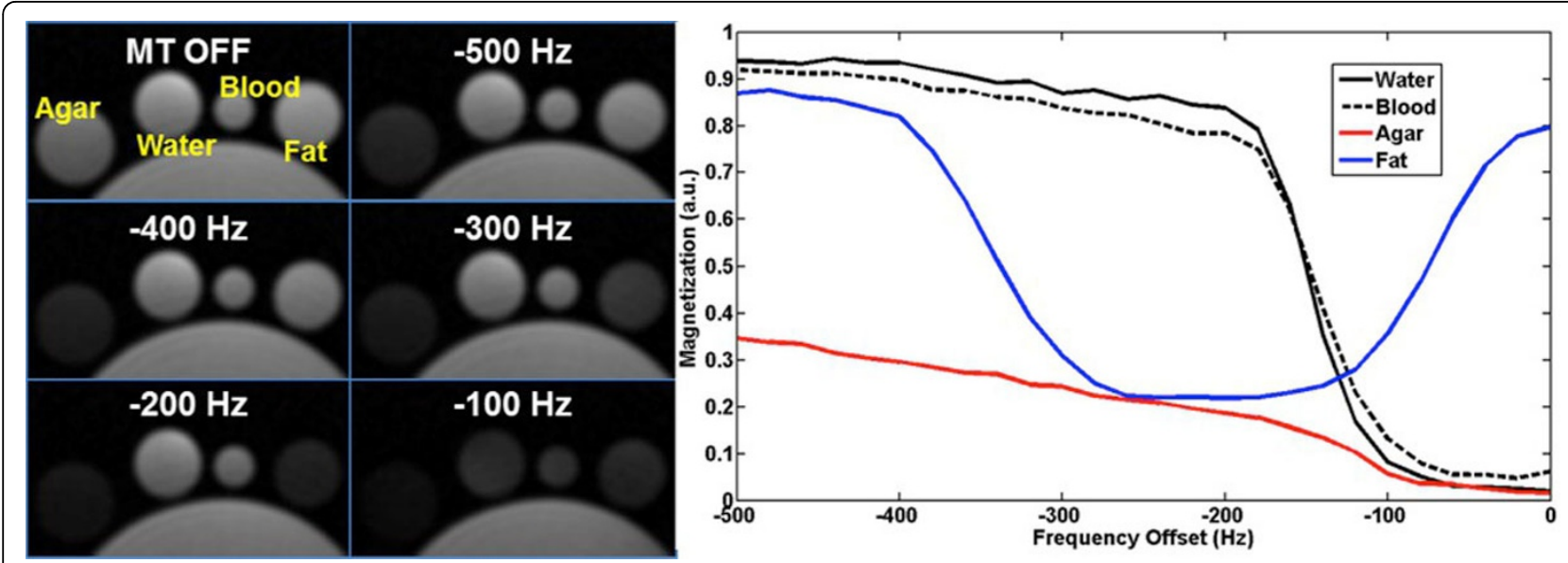

Figure 1 Left: MT-prepared VIPR SPGR scans in phantoms with water, 4\% agar, blood-mimicking fluid, and canola oil (fat) demonstrate signal saturations at various MT offset frequencies. Right: Signal calculations over a range of frequencies show maximum fat suppression near its peak at $1.5 \mathrm{~T}$.

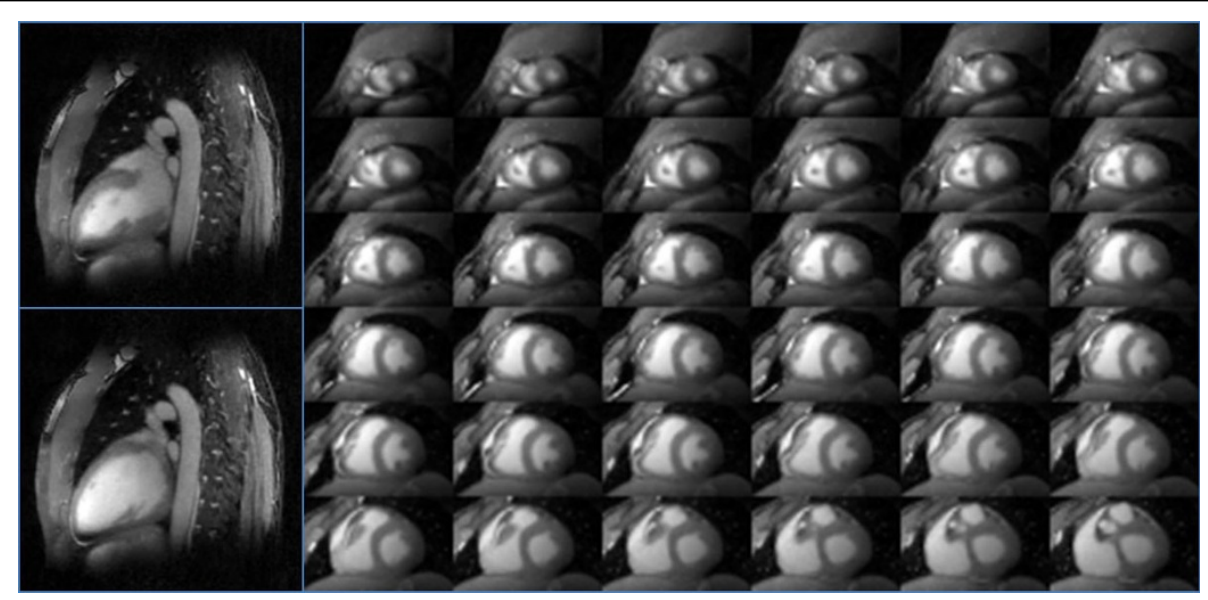

Figure 2 Left: Vertical long axis reformats in end-systole (top) and end-diastole (bottom) display excellent suppression of fat and muscle without off-resonance induced banding artifacts seen in bSSFP. Right: End-systolic short-axis stack from apex to base displays benefits of isotropic spatial resolution for retrospective reformatting of the entire heart in any orientation.

\section{Authors' details}

'Medical Physics, University of Wisconsin - Madison, Madison, Wisconsin,

USA. ${ }^{2}$ Radiology, University of Wisconsin - Madison, Madison, Wisconsin, USA.

Published: 16 January 2014

\section{References}

1. Brittain JH, et al: MRM 1998.

2. Henkelman RM, et al: NMR Biomed 2001.

3. Barger AV, et al: MRM 2000.

\section{doi:10.1186/1532-429X-16-S1-P63}

Cite this article as: Schrauben et al:: Free-breathing 3D cardiac function with accelerated magnetization transfer prepared imaging. Journal of Cardiovascular Magnetic Resonance 2014 16(Suppl 1):P63.

Submit your next manuscript to BioMed Central and take full advantage of:

- Convenient online submission

- Thorough peer review

- No space constraints or color figure charges

- Immediate publication on acceptance

- Inclusion in PubMed, CAS, Scopus and Google Scholar

- Research which is freely available for redistribution 